\title{
Self-Formation and Synchronization of Emitters in Broad Area Lasers in External Cavities
}

\author{
Mark Lichtner ${ }^{1}$, Mindaugas Radziunas ${ }^{1}$, Vasile Tronciu ${ }^{1}$ and Andreas Jechow ${ }^{2}$ \\ ${ }^{1}$ Weierstrass Institute for Applied Analysis and Stochastics, Laser-Dynamics Group, Mohrenstr. 39, 10117 Berlin \\ ${ }^{2}$ Institute for Physics, University of Potsdam, Am Neuen Palais 10, 14469 Potsdam, Germany \\ lichtner@wias-berlin.de
}

\begin{abstract}
Using a new model for dynamic simulation of broad area diode lasers with external cavities we find self formation of synchronized emitters for a broad area laser in a V-shaped external cavity. The results are compared to stripe array diode lasers.

(C)2009 Optical Society of America

OCIS codes: (140.2020) Diode lasers;
\end{abstract}

High power diode lasers are efficient light sources for coherent radiation. Broad area diode lasers (BALs) are capable to produce several Watts of output power at efficiencies above $60 \%$. However, high intensities go hand in hand with a poor beam quality, chaotic behavior and optical field filamentation due to electric or thermal lensing. These problems can be overcome by using striped contacts and, additionally, by locating such a striped array diode laser (SAL) within a V-shaped cavity: see Fig. 1, where a schematic representation of this cavity is shown.

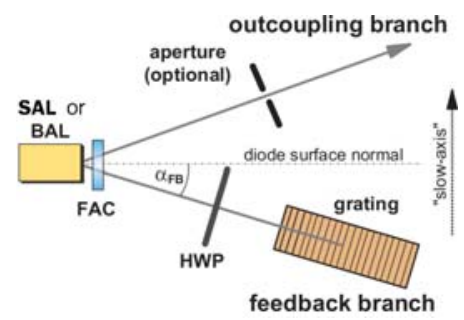

Fig. 1: Setup of the V-shaped cavity containing a broad area or a striped array diode laser, a grating, a half wave-plate (HWP), a fast axis collimator (FAC), and an aperture.

The V-shaped external cavity is adjusted to enhance and stabilize the anti-phase coupling of SALs. Thus, an array can be operated in a single stable high power anti phase supermode. The phase coupling between individual emitters of a stripe-array in an off-axis external resonator results in a narrow bandwidth diffraction limited emission [1]. This opens the door for application of SALs to efficient generation of blue light by second harmonic generation [2]. Recently, a 2+1 dimensional dynamic partial differential equation model [3] was applied for simulation of SALs with external optical feedback from off-axis external cavities [4]. The model is based on a paraxial ray approximation of Maxwell's wave equation for the electric field. Within this approach, along the longitudinal propagation axis counter-propagating slowly varying optical fields are calculated by solving the laterally diffractive traveling-wave equations. The field equations are extended by linear polarization equations describing a Lorenzian profile of the material gain dispersion and are nonlinearly coupled to a dynamic excess carrier density model given by a parabolic diffusion equation. Feedback from the external cavity is given by Fresnel integration.

We have shown in [4] that this model can reproduce, predict and explain experimentally observed near and far field characteristics of SALs depending on the off-axis angle of the external cavity. In the present paper we report numerically observed emitter self organization of BALs in the V-shaped cavity shown in Fig. 1. We compare the results to SALs operated in the same cavity.

Left and right panels of Figs. 2-4 represent near and far fields of a SAL and BAL, respectively. Here, the pitch of the stripes in the SAL was $d=10 \mu \mathrm{m}$, and the off-axis angle of the external cavity in both cases was set to $\alpha_{\mathrm{FB}}=-\alpha$, where $\alpha=\lambda /(2 \mathrm{~d}) \approx 2.8^{\circ}$ ( $\lambda$ : the wavelength of the emitted field).

The left panel of Fig. 2 shows the simulated near field distribution of the SAL. For the SAL the stripes are lasing antiphase. Hence the output beam splits into two main rays radiating at angles $\pm \alpha$. This is observed as two peaks in the far field: see the computed and measured far fields in the left panels of Figs. 3 and 4. The special selection of the feedback angle $\alpha_{\mathrm{FB}}$ supports the antiphase coupling of the stripes. Thus a stable optical supermode with intensity maxima located within each stripe (upper left panel of Fig. 2) is supported. As can be seen in the lower left panel phases between neighboring stripes differs by $\pi$. 


\section{JTuD100.pdf}

In contrast, the BAL is fully contacted and does not radiate at $\pm \alpha$ by its own. When putting the BAL into the same cavity, as can be seen in the simulated near field in the right top panel of Fig. 2, the BAL forms emitters (periodical field maxima) for positive lateral coordinates. The distance between field maxima is precisely $d$. The self-formed emitter distance $d$ can be tuned by adjusting the feedback angle $\alpha_{\mathrm{FB}}$. While the SAL is stabilized mainly by gain guiding the stability of the self formed emitters in the BAL can be explained by electronic self-focusing and feedback.

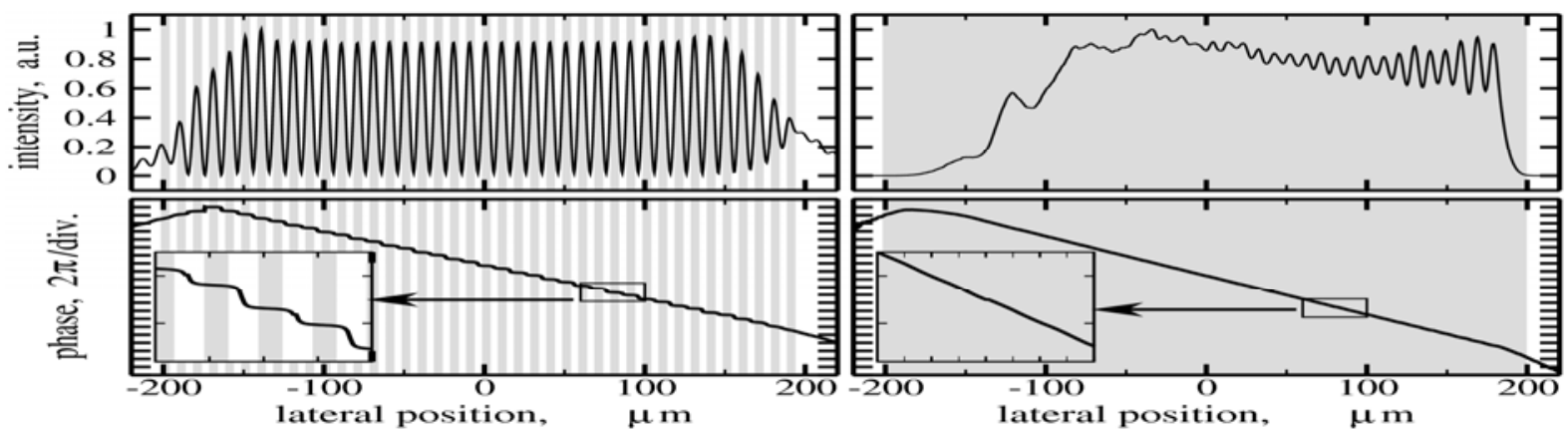

Fig. 2: Calculated near-field distribution (top) and phases (bottom) for a stripe array (left) and a BA diode laser (right) with off-axis external feedback. Shaded regions show location of stripes or contact.

According to the phases shown in the lower right panel of Fig. 2, neighboring field maxima are anti-phase synchronized similarly as in the stripe array. However, in contrast to the SAL, which has a lateral step-like change of the optical field phase (lower left panel of Fig. 2), the near-field phase of the BAL rotates continuously with the same averaged slope determined by the cavity angle $\alpha$. This nearly linear change of the near field phase, which corresponds mainly to a single outgoing plane wave, is represented also by the theoretical far field (right panel of Fig. 3) with an almost complete suppression of the feedback lobe at the angle $-\alpha$. The experimental far field of the BAL shown in the right panel of Fig. 4 is wider and has a broad lobe around $-\alpha$. This could be due to emitter desynchronization caused by thermal defocussing which has not been considered in our modeling.

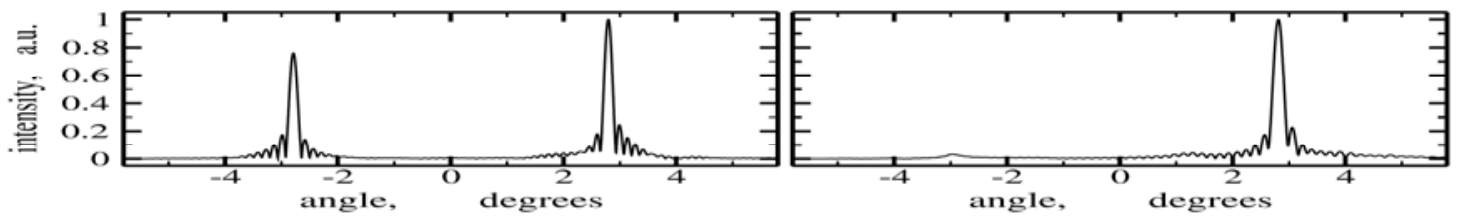

Fig. 3: Calculated far-field distribution of the stripe array (left) and BA diode laser (right) with off-axis external feedback
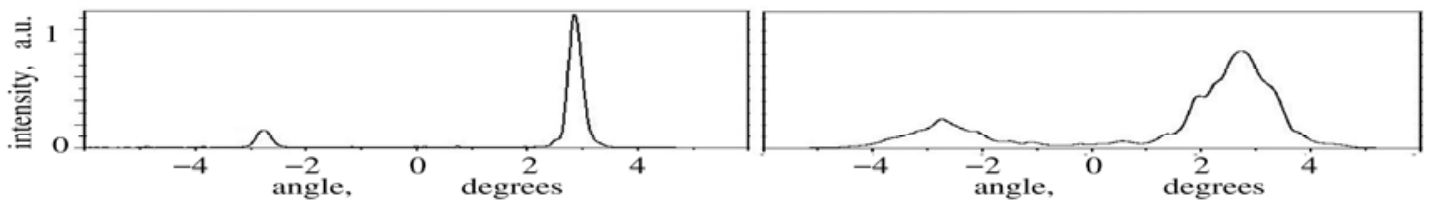

Fig. 4. Measured far-fields for the stripe array (left) and the BA diode laser (right) with off-axis external feedback.

In conclusion, our numerical simulations have shown that through a self-organization BALs in V-shaped cavities form anti-phase synchronized stripe arrays. Hence, similarly as for stripe contacted devices, a plane wave resonator is formed [4].

\section{References}

[1] A. Jechow, V. Raab, R. Menzel, M. Cenkier, S. Stry and J. Sacher, "1 W tunable near diffraction limited light from a broad area laser diode in an external cavity with a line width of $1.7 \mathrm{MHz}$, Optics Communications 277, 161-165 (2007)

[2] A. Jechow and R. Menzel, "Efficient blue light generation by frequency doubling of a broad-area diode laser in a compact external cavity," Applied Physics B 89, 507-511 (2007)

[3] M. Spreemann, M. Lichtner, M. Radziunas, U. Bandelow, and H. Wenzel, "Measurement and Simulation of Distributed-Feedback Tapered Master-Oscillator Power Amplifiers," IEEE J. Quantum Electron. 45, 609-616 (2009).

[4] A. Jechow, M. Lichtner, R. Menzel, M. Radziunas, D. Skoczowsky, and A. G. Vladimirov, "Stripe-array diode-laser in an off-axis external cavity: Theory and experiment," Opt. Express 17, 19599-19604 (2009) 